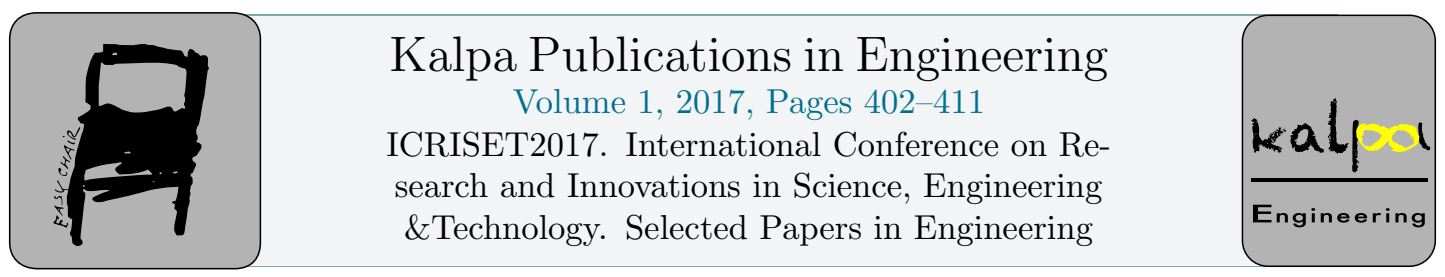

\title{
Automatic Image Registration
}

\author{
Dr. Mehfuza Holia ${ }^{1}$ \\ * Prof. Zankhana Shah ${ }^{2}$ \\ ${ }^{1}$ Electronics Dept \\ ${ }^{2}$ Computer Dept, BVM Engineering College \\ msholia@bvmengineering.ac.in, zankhana.shah@bvmengineering.ac.in
}

\begin{abstract}
The automatic construction of large, high-resolution multi view image registration is an active area of research in the fields of image processing. Multiview image registration can be used for many different applications. The most traditional application is the construction of large aerial and satellite photographs from collections of images, construction of virtual travel etc. This proposed Automatic feature based image registration method does not allow any user interaction and perform all registration steps automatically. Here the matching points are found automatically using local feature detector i.e. harris corner detector which find invariant features using feature descriptors as oriented patches. For estimating homography between detected features of images to be registered, Homography estimator i.e. modified RANSAC (RANdom SAmple Consensus) algorithm, and direct linear transformation algorithm is used. Here features are located at Harris corners (new improved) in discrete scale-space and oriented using a blurred local gradient. To have better spatial distribution of features, adaptive nonmaximal suppression algorithm is used.Feature matching are achieved using RANSAC which also uses DLT (Direct Linear Transformation) and warping is applied to achieve final registered image. This proposed algorithm can be applied for the series of images that may or may not be in the same alignment as per desired output image, thus mainly scaling, rotation and image transformation must be applied to get proper registered image.
\end{abstract}

\section{Introduction}

Image Registration is a important step in all image processing application where the final output information is gained from the combination of various image sources. The geometric alignment of reference and sensed images has been done by image registration. The present differences between images can be because of different imaging sensors, detection technique and image restoration.

* Masterminded EasyChair and created the first stable version of this document 
Registration is required in remote sensing for mosaicking, multi view images, monitoring of environment, detection of changes, forecasting, creating super-resolution images, medical applications etc, and has many features to make it suitable for various conference models. It is currently probably the

\section{Interest point detection}

From the literature [7,8], it has been concluded that methods based on intensity, when compared in stability and speed of each algorithm, harris corner detection algorithm is superior to other corner detection algorithm. Thus for finding the interest points, multi-scale harris corner detector is used. In our research unlike haris corner detector, we have not consider user detected corner points for accurate point selection and function of cornerness is also modified to give more accuracy then harris corner response. Harris corner detection algorithm is realized by calculating each pixel's gradient. If the absolute gradient values in two directions are both great, then judge the pixel as a corner.

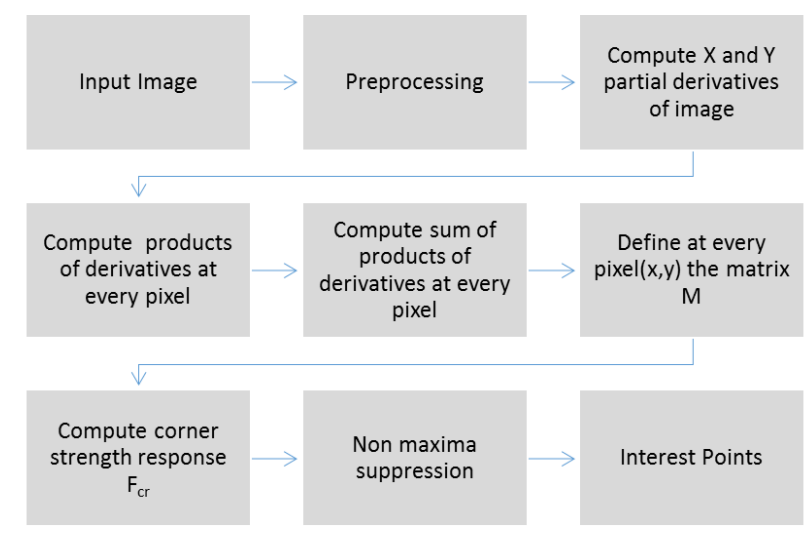

\section{Fig.1 Flowchart for interest point corner detectors}

\section{Extraction of Feature Descriptor}

After determination of interest points, the description of the local image structure is extracted for reliable and efficient matching of features across images to be registered. Given an interest point (x, y) the descriptor is formed by a patch centered at $(\mathrm{x}, \mathrm{y})$. We sample $21 \mathrm{x} 21$ patch of pixels around the subpixel location of the interest point. Now these extracted patch descriptors, around detected local features descriptors are blurred by a (fairly large) Gaussian with sigma=3 pixels. So for interest point $(\mathrm{x}, \mathrm{y})$ the patch is given by

$$
\mathrm{P}=\mathrm{I}(\mathrm{x}-\mathrm{w}: \mathrm{x}+\mathrm{w}, \mathrm{y}-\mathrm{w}: \mathrm{y}+\mathrm{w}) \quad \text { where } \mathrm{w}=10 \text { for patch of } 21 \mathrm{x} 21 .
$$




\section{Matching of local features}

After finding the descriptors of images to be registered, the tentative matches are computed by matching descriptor. It is required to find nearest neighbors for all features from all images, which is the well known all nearest neighbors problem. To match the descriptors, euclidean distance between descriptors are found. Suppose if Da is descriptors of image Ia and Db is descriptor of image Ib, where $\mathrm{Da}$ and $\mathrm{Db}$ are of size MxN1 and MxN2 respectively (transpose be-comes size of N1 x M and N2 x $\mathrm{M})$, then Euclidean distance between them is given by

$$
D_{E}\left(N_{1}, N_{2}\right)=\sum_{i=1}^{N_{1}} \sum_{j=1}^{N_{2}}\left[D_{a}{ }^{\prime}(i,:)-D_{b}{ }^{\prime}(j,:)\right]^{2}
$$

To reduce computation load, we have taken only those descriptors which is having minimum Euclidean distance. Thus DE is shorted in terms of minimum Euclidean distance. To obtain distinctive features, we use the ratio of distances to the first and the second nearest neighbour, which has been taken 1 (i.e. below 0.8 ) so that the best match (1st nearest neighbour) should be much closer than the next best match (the 2nd nearest neighbour). Here only those interest points are selected from both images to be registered, whose descriptors are same or having minimum euclidean distance, so as a result we can have tentative matches between Image Ia and Ib.

\section{Homography Estimation}

A homography, which is a projective transformation to map points in one plane to another plne. In our case, the planes are images. In essence, a homography $\mathrm{H}$ maps $2 \mathrm{D}$ points according to

$$
\left[\begin{array}{c}
x^{\prime} \\
y^{\prime} \\
w^{\prime}
\end{array}\right]=\left[\begin{array}{lll}
h_{1} & h_{2} & h_{3} \\
h_{4} & h_{5} & h_{6} \\
h_{7} & h_{8} & h_{9}
\end{array}\right]\left[\begin{array}{c}
x \\
y \\
w
\end{array}\right]
$$

OR

$$
X^{\prime}=H X
$$

Points in homogeneous coordinates are only defined up to scale so that $\mathrm{X}=[\mathrm{x}, \mathrm{y}, \mathrm{w}]=[\alpha \mathrm{x}, \alpha \mathrm{y}, \alpha \mathrm{w}]=$ $[\mathrm{x} / \mathrm{w}, \mathrm{y} / \mathrm{w}, 1]$, all refer to the same $2 \mathrm{D}$ point. Normalization of points has been done with $\mathrm{w}=1$ to have a unique identification of the image coordinates $\mathrm{x}, \mathrm{y}$. We can represent transformation using single matrix as given in equation (2).

The RANdom SAmple Consensus (RANSAC) algorithm proposed by Fischler and Bolles[48] is a method for general parameter estimation. It is a resampling method for generating solutions which uses the minimum number observations for estimation of the underlying model parameters. It uses the smallest set possible and proceeds to enlarge this set with consistent data points [8]. The data are classifies into into inliers (valid points) and outliers and estimate the optimal transformation of inliers. For a number of iterations, a random sample of 4 correspondences is selected and a homography $\mathrm{H}$ is computed from those four correspondences. Each other correspondence is then classified as an inlier or outlier depending on its concurrence with $\mathrm{H}$. After the completion of all the iterations, the iteration that contained the largest number of inliers is selected, then $\mathrm{H}$ can then be recomputed from all of the correspondences that were considered as inliers in that iteration. In our research we have used modified RANSAC algorithm to estimate robust homography. 
Following steps are followed to achieve 2D homography between images to be registered using modified RANSAC.

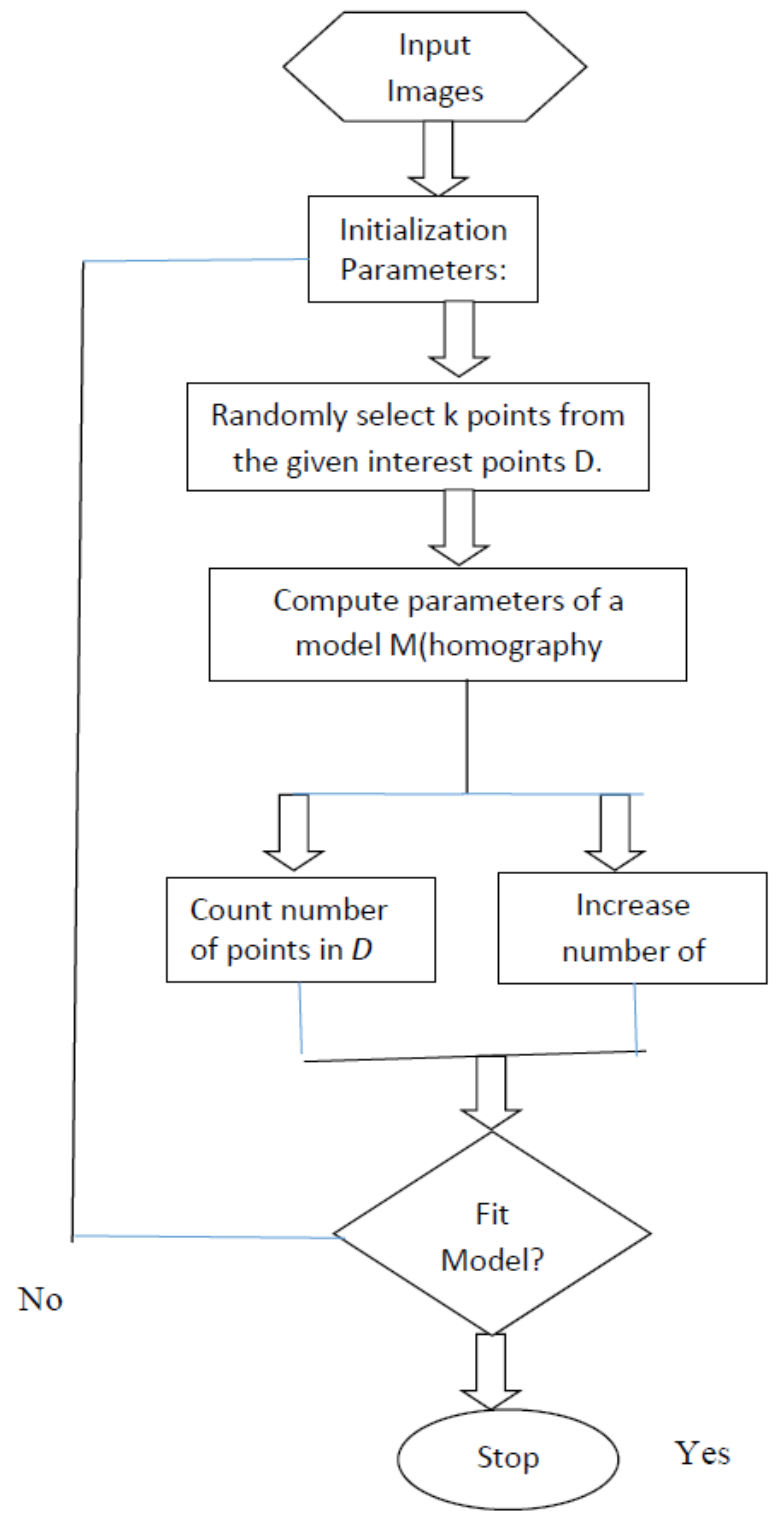

Fig.2 Flow chart for computing 2D homography. 


\section{Image Warping and Re sampling Using Bilinear Interpolation}

Image warping is a transformation which maps all positions in one image plane to positions in a second plane. To change the spatial configuration of an image, Image warping is required..

In bilinear interpolation technique, the new intensity is determined from the weighted sum of intensities at four pixels closest to it. Therefore, given location $(X, Y)$ and assuming a is the integer part of $X$ and $b$ is the integer part of $Y$, the intensity at $(X, Y)$ is estimated from the intensities at $(a, b)$, $(a+1, b),(a, b+1),(a+1, b+1)$. This can be summarized as

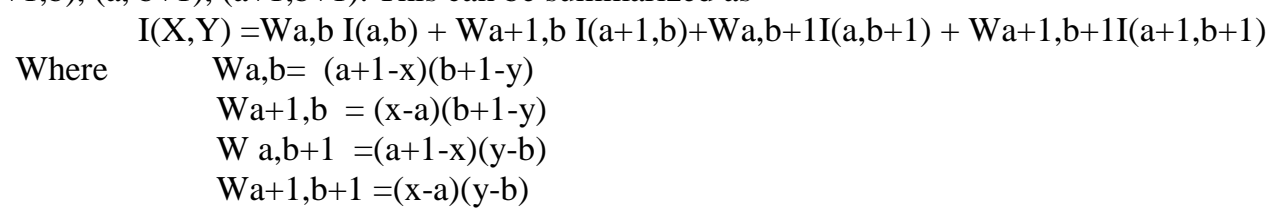

Here warping returns an interpolated warp of image under homography. Warping is done on the images using the estimated homography. Here backward mapping is used so for that first it is necessary to define a registered image (in terms of index) to warp all the images onto, so second image will be taken as reference image and map this image to the origin of the registered image using the identity homography. Now first image will be registered with second using homography matrix and bilinear interpolation.

\section{Proposed Algorithm}

Input : Images A and B (or more than two) of size $\mathrm{M} \times \mathrm{N}$ to be registered

Output: Registered Image

1. Apply Gaussian Smoothing for each input image I(x,y)

2. Detect interest points using local feature as explained in section II

3. Apply Non maxima suppression to select only spatially well distributed points over the

4. Define Feature descriptors for selected interest points for both images as described in section III

5. Match the Local features using Euclidean distances using methods described in section IV

6. Estimate Homography using modified RANSAC, Direct linear transformation(DLT) and Singular Value Decomposition (SVD) as described in section V VI.

7. Apply Image Warping using backward mapping and interpolation techniques as stated in section

8. Registered output image.

9. Repeat steps 1 to 8 for registration of more than two images .

10. End

\section{Results}

Experimental results are carried out on different multiview images. Here no selection points are required. Following table 6.1 shows the $\mathrm{N}$ iteration for different interest point and different probability of observing an outlier v. As described earlier, in this proposed algorithm we have taken $\mathrm{p}=0.99$, that at least one of the sets of random samples does not include an outlier. 
Let $\mathrm{u}$ represent the probability that any selected data point is an inlier and $\mathrm{v}=1-\mathrm{u}$ the probabil-ity of observing an outlier. Ninl is number inliers which satisfies the threshold criteria. $\mathrm{N}$ iterations of the minimum number of points denoted s (minimum No of points needed to fit a homography) are required, as given in equation (5)

$$
\mathrm{N}=\frac{\log (1-\mathrm{p})}{\log \left(1-(1-\mathrm{v})^{\mathrm{s}}\right)}, \mathrm{w}=1-\mathrm{v}=1-\frac{\mathrm{N}_{\text {inl }}}{\mathrm{m}}
$$

TABLE I : VALUE OF REQUIRED ITERATION FOR DIFFERENT VALUE OF S AND W=0.95

\begin{tabular}{|l|c|c|c|c|}
\hline $\begin{array}{l}\text { Probability } \\
\text { w }\end{array}$ & S & $\mathbf{N}$ & $\begin{array}{c}\text { N > } \\
\text { current } \\
\text { iteration } \\
\text { count }\end{array}$ & $\begin{array}{c}\text { Further } \\
\text { iterations } \\
\text { required }\end{array}$ \\
\hline \multirow{4}{*}{0.95} & 2 & 2 & Yes & Yes \\
\cline { 2 - 5 } & 2 & 2 & No & No \\
\cline { 2 - 5 } & 3 & 3 & Yes & Yes \\
\cline { 2 - 5 } & 3 & 3 & No & No \\
\cline { 2 - 5 } & 4 & 3 & Yes & Yes \\
\cline { 2 - 5 } & 4 & 3 & No & No \\
\cline { 2 - 5 } & 5 & 4 & Yes & Yes \\
\cline { 2 - 5 } & 5 & 4 & No & No \\
\cline { 2 - 5 } & 6 & 4 & Yes & Yes \\
\cline { 2 - 5 } & 6 & 4 & No & No \\
\cline { 2 - 5 } & 7 & 4 & Yes & Yes \\
\cline { 2 - 5 } & 7 & 4 & No & No \\
\cline { 2 - 5 } & 8 & 5 & Yes & Yes \\
\cline { 2 - 5 } & 8 & 5 & No & No \\
\hline
\end{tabular}

TABLE II :VALUE OF OF REQUIRED ITERATION N FOR DIFFERENT VALUE OF S AND W

\begin{tabular}{|c|c|c|c|c|c|c|c|}
\hline $\mathbf{s}$ & $\begin{array}{c}\mathbf{W}= \\
\mathbf{0 . 9 5}\end{array}$ & $\begin{array}{c}\mathbf{W}= \\
\mathbf{0 . 9 0}\end{array}$ & $\begin{array}{c}\mathbf{W}= \\
\mathbf{0 . 8 0}\end{array}$ & $\begin{array}{c}\mathbf{W}= \\
\mathbf{0 . 7 5}\end{array}$ & $\begin{array}{c}\mathbf{W = 0} \\
\mathbf{. 7 0}\end{array}$ & $\begin{array}{c}\mathbf{W}= \\
\mathbf{0 . 6 0}\end{array}$ & $\begin{array}{c}\mathbf{W = 0} \\
\mathbf{. 5 5}\end{array}$ \\
\hline 2 & 2 & 3 & 5 & 6 & 7 & 11 & 17 \\
\hline 3 & 3 & 4 & 7 & 9 & 11 & 19 & 35 \\
\hline 4 & 3 & 5 & 9 & 13 & 17 & 34 & 72 \\
\hline 5 & 4 & 6 & 12 & 17 & 26 & 57 & 146 \\
\hline 6 & 4 & 7 & 16 & 24 & 37 & 97 & 293 \\
\hline 7 & 4 & 8 & 20 & 33 & 54 & 163 & 588 \\
\hline 8 & 5 & 9 & 26 & 44 & 78 & 272 & 1177 \\
\hline
\end{tabular}

So from table 1 and 2, we can conclude that as the probability for inliers is more, the required iterations are less. Threshold for selecting homography matrix $=0.01$, which gives us maximum registration accuracy in terms of standard deviation and entropy compared to already registered image taken from camera. We have tried this for more than 40 images and it gives us best result. 

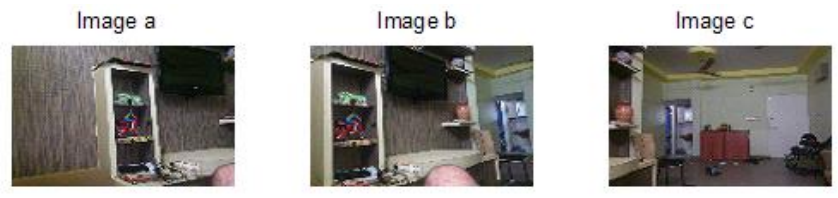

Fig.3 Input images

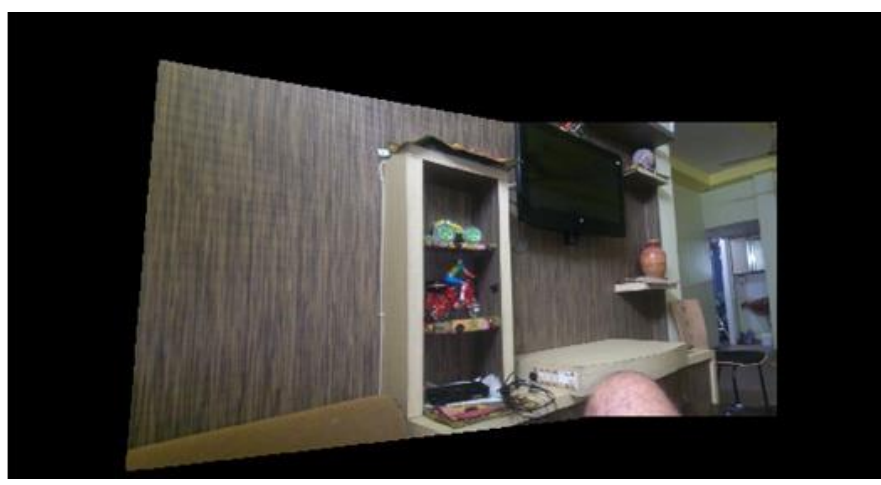

(a)

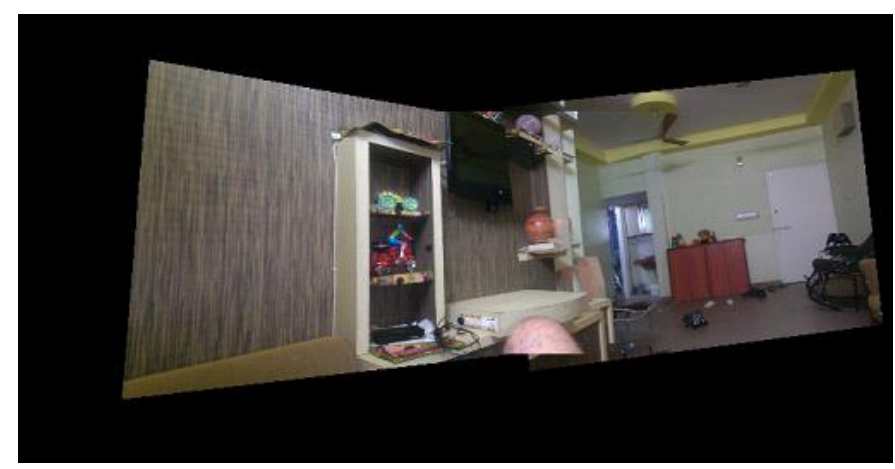

Fig 4 Registration of two (a) and three (b) images

Imoge a

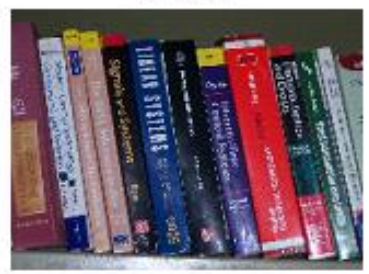

Image b

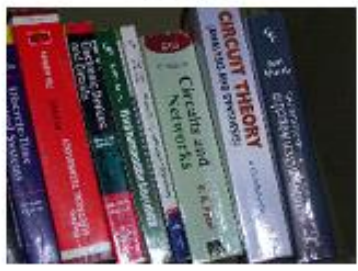

Fig 5 Input images 


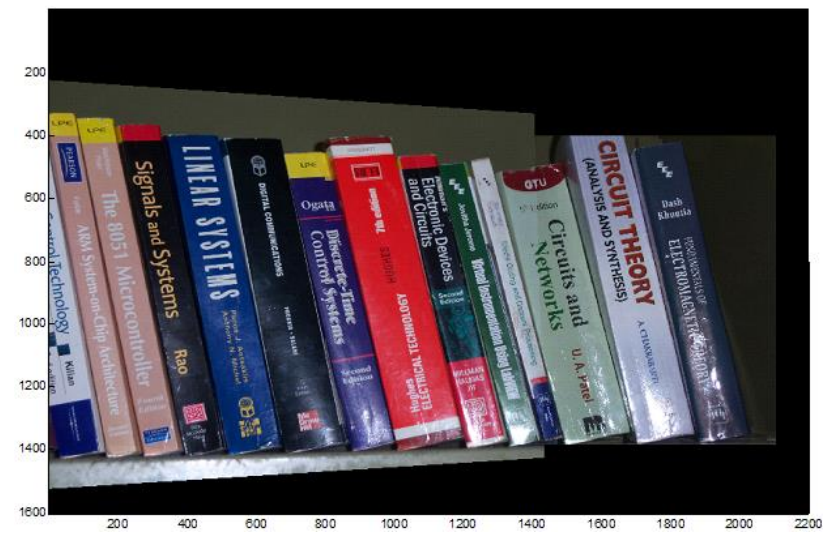

Fig. 6 Registered image

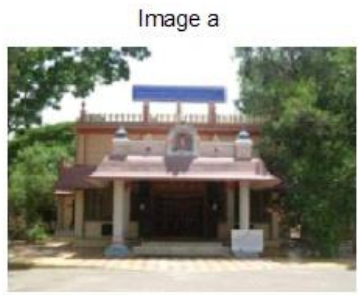

(a)

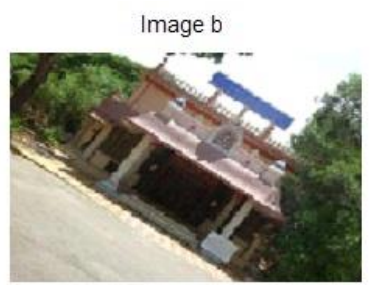

(b)

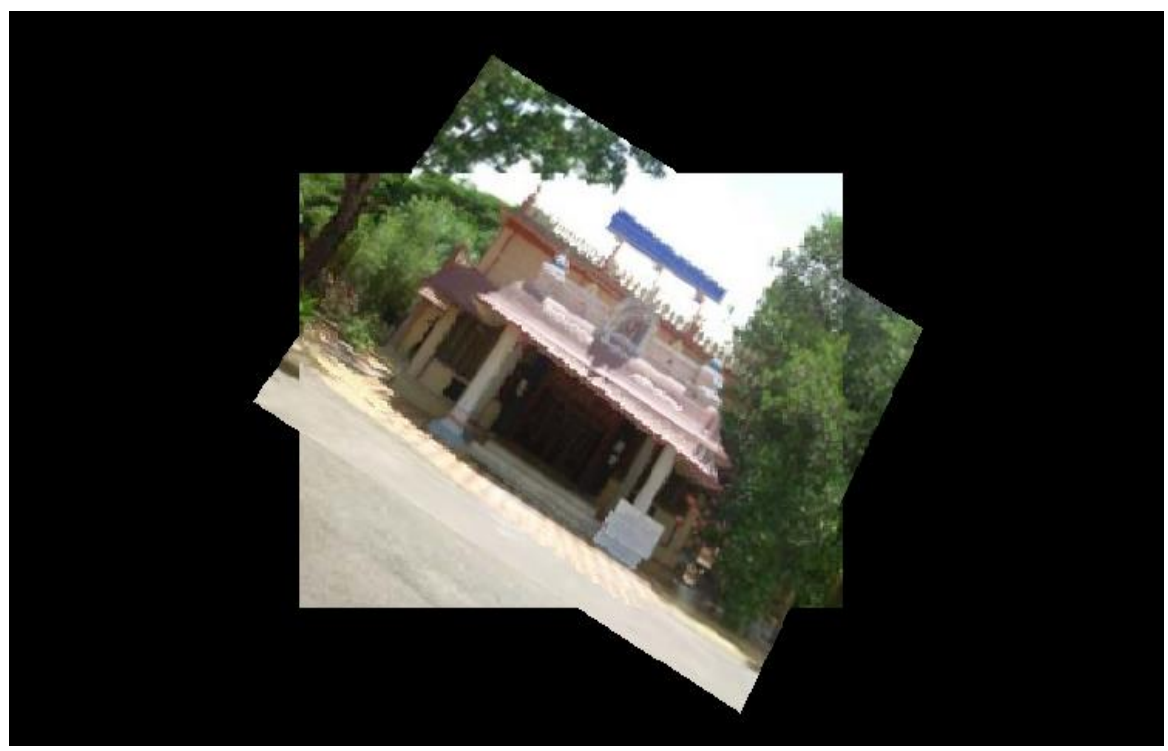

(c)

Fig.7 Input images (a-b) and registered image(c) 


\section{Conclusions}

This Paper has presented an algorithm for fully automatic multiview image registration. Here the invariant local features and a probabilistic model for homography have been used to verify image matches to register them fully automatically without user input. This algorithm is robust to camera zoom, orientation of the input images to be registered and changes in illumination. A blending scheme ensures smooth transitions between images despite illumination differences, while preserving high frequency details.

In this proposed algorithm, after detecting the interest points, we have also implemented non maximal suppression that better distributes features across the image so it will improve matching with less overlap We have tested this algorithm for 40 images and success ratio is $100 \%$. By taking many multiview images, we can significantly increase the field of view of the resultant registered images and remove the need for expensive fisheye lenses.

\section{References}

[1]Y.Bentoutou,N.Taleb,K.Kpalma,J.Ronsin, “An automatic image registration for appl- ications in remote sensing." IEEE transactions on geoscience and remote sensing ,43(9) (2005):3137-3137.

[2]Jing Zhang, Ying Shan,Mao-Zu Guo, “An edge-detection method for moving objects” Proceeding of IEEE International Conference on Automation and Logistics Jinan, China .pages 1557-1561.18-21 August 2007.

[3]Harrywechsler,Masatsugukidode,"A new edge detection technique and its implemen- tation" IEEE transaction on systems man and cybernetics,7(12)(1977):827-837.

[4]Kefeng fan Qingqi,Pei Wei Mo,Xinhua Zhao,Qifeng Sun,“An efficient automatic iris image acquisition and preprocessing system",Proceeding of IEEE International Confer-ence on Mechatronics and Automation,China. Pages 1779 -1784, 25-28 June 2006.

[5]Imam S,Amil Yetik,A. Nehorai,“ Performance bounds on image registration”, IEEE transactions On Signal Processing, 54(5)(2006):1737-1750.

[6]Zhao Yu-qian ,Gui Wei-hua,Chen Zhen-cheng,Tang Jing-tian, Li Ling-yun, "Medical images edge detection based on mathematical morphology", Proceeding IEEE 27thAnnual conference on Engineering in Medicine and Biology.China, pages 6492-6495,September 1-4,2005.

[7]Jie Chen,Li-Hui Zou,Juan Zhang,Li-Hua Dou,'The comparison and application of corner detection algorithms", Journal Of Multimedia, 4(6)(2009):435-441.

[8]D.Parks,J.P.Gravel,“lecture notes on corner detection”-2011.

[9]Angus M. K. Siu,Rynson,W.H. Lau,'Image registration for image-based rendering" IEEE transactions On Image Processing,14(2),(2005):241-253.

[10]J Osien P.W.Pluim,J.M Ichael Fitzpatrick" Image registration", IEEE transactions on medical imaging,22(11)(2003):1341-1344.

[11]M.S.Holia, V.K.Thakar, "Survey of image registration methods" Journal of Engineering \& Technology (GIT-JET),2(2009):64-71

[12]M.S.Holia,V.K.Thakar," Image registration for recovering affine transformation using Nelder Mead simplex method for optimization" International Journal of Image Processing IJIP), Computer Science Journals, 3(5)(2010):251-256 
[13]M.S.Holia,H.R.Kher V.K.Thakar, Registration of license plate images captured at different angles using mutual information ",2011.Proceeding of IEEE International Conference on com-putational Intelligence and computing research Dec 15-18, 2011.

[14]M.S.Holia,V.K.Thakar,"Mutual information based image registration for MRI and CT scan brain images", Proceeding of IEEE International Conference on Audio, Language and Image Processing. July $16-18,2012$

[15].Mehfuza Holia,"Multi View Image Registration” , Springer Publication (SIST)-ICTIS-2015, November 2015. 